\title{
On finite commutative IP-loops with elementary abelian inner mapping groups of order $p^{5}$
}

\author{
Markku Niemenmaa
}

\begin{abstract}
We show that finite commutative inverse property loops with elementary abelian inner mapping groups of order $p^{5}$ are centrally nilpotent of class at most two.
\end{abstract}

Keywords: Loop, elementary abelian group, inner mapping group

Mathematics Subject Classification: 20D10, 20N05

\section{Introduction}

If $Q$ is a loop then the mappings $L_{a}(x)=a x$ and $R_{a}(x)=x a$ are called the left and right translation. These two mappings are permutations on $Q$ for every $a \in Q$ and the permutation group $M(Q)=\left\langle L_{a}, R_{a}: a \in Q\right\rangle$ is called the multiplication group of $Q$. The stabilizer of the neutral element of $Q$ is the inner mapping group of $Q$ and we denote it by $I(Q)$. If $Q$ is a group then $I(Q)=\operatorname{Inn}(Q)$, the group of inner automorphisms of $Q$.

The centre $Z(Q)$ of a loop $Q$ contains all elements $a$ with the property that $a x=x a,(a x) y=a(x y),(x a) y=x(a y)$ and $(x y) a=x(y a)$ for every $x, y \in Q$. The centre $Z(Q)$ is an abelian group and if we write $Z_{0}=1, Z_{1}=Z(Q)$ and $Z_{i} / Z_{i-1}=Z\left(Q / Z_{i-1}\right)$, then we have a series of normal subloops of $Q$. If $Z_{n-1}$ is a proper subloop of $Q$ and $Z_{n}=Q$, then $Q$ is said to be centrally nilpotent of class $n$. Bruck [1] showed that if $Q$ is centrally nilpotent of class at most two, then $I(Q)$ is an abelian group. Csörgö [3] showed that the converse of Bruck's result is not true by constructing a centrally nilpotent loop $Q$ whose nilpotency class is three and whose inner mapping group $I(Q)$ is an elementary abelian group of order $2^{6}$. More examples and constructions of of loops with nilpotency class three and elementary abelian inner mapping groups of order $2^{6}$ were given by Drápal and Vojtěchovský [4]. Earlier results by Csörgö, Kepka and Niemenmaa (see [2] and [6]) cover the cases where $I(Q)$ is elementary abelian of order $p^{2}$ and $p^{3}$ and it turned out that $Q$ is then centrally nilpotent of class at most two. 
A loop $Q$ is an inverse property loop (in short, IP-loop) if $Q$ has a unique left and right inverse $x^{-1}$ and $x^{-1}(x y)=y=(y x) x^{-1}$ for every $x, y \in Q$. Niemenmaa [9] proved that if $Q$ is a finite commutative IP-loop and $I(Q)$ is elementary abelian of order $p^{4}$, then $Q$ is centrally nilpotent of class at most two. The purpose of this paper is to show that in the case of finite commutative inverse property loops, the nilpotency class is also at most two provided that the inner mapping group is elementary abelian of order $p^{5}$.

We consider only finite loops and groups in this paper. The proofs of our main theorems rely on the use of connected transversals in finite groups and this notion and some basic results about these transversals are explained in the following section. For basic facts about loop theory and its connections to group theory the reader is advised to consult [1] and [5].

\section{Connected transversals}

We shall start with a brief discussion about connected transversals in a group and try to give some insight into the relationship between loops and groups given by this notion.

Let $G$ be a group and $H \leq G$. If $A$ and $B$ are two left transversals to $H$ in $G$ and $a^{-1} b^{-1} a b \in H$ for every $a \in A$ and for every $b \in B$, then we say that the two transversals are $H$-connected in $G$. If $A=B$, then we say that $A$ is a selfconnected transversal to $H$ in $G$. In the following lemmas and theorems we consider some basic properties of $H$-connected transversals $A$ and $B$. We denote by $H_{G}$ the core of $H$ in $G$ (it is the largest normal subgroup of $G$ contained in $H)$.

Lemma 2.1. If $C \subseteq A \cup B$ and $K=\langle H, C\rangle$, then $C \subseteq K_{G}$.

For the proof, see [5, Lemma 2.5].

Lemma 2.2. If $H_{G}=1$, then $N_{G}(H)=H \times Z(G)$.

For the proof, see [5, Proposition 2.7].

Theorem 2.3. Let $H$ be a nilpotent subgroup of $G$. If $G=\langle A, B\rangle$ and $H_{G}=1$, then $H$ is subnormal in $G$ and $Z(G)>1$.

For the proof, see [8, Theorem 2.8].

Theorem 2.4. If $H$ is cyclic and $G=\langle A, B\rangle$, then $G^{\prime} \leq H$.

For the proof, see [5, Theorem 3.5].

Theorem 2.5. Let $p$ be a prime number. If $H \cong C_{p} \times C_{p}$ and $G=\langle A, B\rangle$, then $G^{\prime} \leq N_{G}(H)$.

For the proof, see [6, Lemma 4.2].

Theorem 2.6. Let $p$ be a prime number. If $H \cong C_{p} \times C_{p} \times C_{p}$ and $G=\langle A, B\rangle$, then $G^{\prime} \leq N_{G}(H)$. 
For the proof, see [2, Theorem 3.7].

Theorem 2.7. Let $H$ be an elementary abelian subgroup of order $p^{4}$ of $G$ and let $A$ a be selfconnected transversal to $H$ in $G$. If $G=\langle A\rangle$ and $A=A^{-1}$, then $G^{\prime} \leq N_{G}(H)$.

For the proof, see [9, Theorem 3.1].

Lemma 2.8. Let $G=\langle A, B\rangle$. If $H$ is nilpotent and $H_{G}=1$, then the core of $H Z(G)$ in $G$ properly contains $Z(G)$.

For the proof, see [10, Lemma 2.6].

Lemma 2.9. Let $H$ be a nontrivial subgroup of $G, H_{G}=1$ and $G=\langle A, B\rangle$. Then $H \cap H^{a}>1$ for every $a \in A \cup B$.

For the proof, see [7, Lemma 2.8].

We shall conclude this section by establishing the relation between connected transversals and loop theory. If $A=\left\{L_{a}: a \in Q\right\}$ and $B=\left\{R_{a}: a \in Q\right\}$ are the sets of left and right translations, then $A$ and $B$ are $I(Q)$-connected transversals in $M(Q)$. Since $M(Q)$ is transitive on $Q$, it follows that the core of $I(Q)$ in $M(Q)$ is trivial. Kepka and Niemenmaa proved the following theorem in 1990 [5, Theorem 4.1].

Theorem 2.10. A group $G$ is isomorphic to the multiplication group of a loop if and only if there exist a subgroup $H$ of $G$ satisfying $H_{G}=1$ and $H$-connected transversals $A$ and $B$ such that $G=\langle A, B\rangle$.

If $Q$ is a commutative loop, then $A=B$. Furthermore, if $Q$ is a commutative inverse property loop, then $\left(L_{a}\right)^{-1}=L_{a^{-1}}$ and thus $A=A^{-1}$.

\section{Main Theorems}

In this section we consider the situation that $A=B, A=A^{-1}$ and $H$ is an elementary abelian group of order $p^{5}$. We first introduce the following two lemmas.

Lemma 3.1. If $H_{G}=1$, then $1 \in A$ and $Z(G) \subseteq A$.

For the proof, see [5, p. 113] and [9, Lemma 2.3]

Lemma 3.2. If $a b=c h$, where $a, b, c \in A$ and $h \in H$, then $h \in H \cap H^{a} \cap H^{b}$.

Proof. Now $h=c^{-1} a b$ and $h^{a^{-1}}=\left(c^{-1} a b\right)^{a^{-1}}=a c^{-1} a^{-1} c c^{-1} a b b^{-1} a b a^{-1} \in H$. We also get $h^{b^{-1}}=\left(c^{-1} a b\right)^{b^{-1}}=b c^{-1} b^{-1} c c^{-1} b a=b c^{-1} b^{-1} c c^{-1} a b h_{1} \in H$ (here $\left.h_{1} \in H\right)$. Thus $h \in H \cap H^{a} \cap H^{b}$.

Theorem 3.3. Let $H$ be an elementary abelian subgroup of a finite group $G$ and let $H$ be of order $p^{5}$. If $A$ is a selfconnected transversal to $H$ in $G, G=\langle A\rangle$ and $A=A^{-1}$, then $G^{\prime} \leq N_{G}(H)$. 
Proof. We shall prove the theorem by induction on the order of $G$. From Theorems 2.4, 2.5, 2.6 and 2.7 it follows immediately that $H_{G}=1$. By Lemma 2.2, $N_{G}(H)=H \times Z(G)$ and $Z(G)>1$ by Theorem 2.3. By Lemma 2.8 the core of $H Z(G)$ in $G$ is equal to $K Z(G)$, where $1<K \leq H$.

If $|K| \geq p^{4}$, then we conclude by Theorem 2.4 that $G^{\prime} \leq H Z(G)=N_{G}(H)$. Thus we may assume that $|K|=p$ or $|K|=p^{2}$ or $|K|=p^{3}$. By applying Theorems 2.4 - 2.7 and Lemma 2.2 on $G / K Z(G)$ and $H Z(G) / K Z(G)$ it follows that $G^{\prime} \leq N_{G}(H Z(G))=H M$. Here $M / K Z(G)=Z(G / K Z(G)), M$ is normal in $G$ and $M \cap H Z(G)=K Z(G)$. We shall now divide the proof into three parts depending on the order of $K$ :

1) Let $|K|=p$. Now we can proceed exactly in the same way as in part 1) of the proof of Theorem 3.1 in [9].

2) Now assume that $K \cong C_{p} \times C_{p}$. Let $a, b \in A$ and $a b=c h$, where $c \in A$ and $h \in H$. If $d \in A$, then $h^{d}=\left(c^{-1} a b\right)^{d}=h_{1} c^{-1} a h_{2} b h_{3}=h_{1} h b^{-1} h_{2} b h_{3} \in H H^{b} H$. As $H Z(G)$ is normal in $H M$ and $H^{b} \leq H M$, we have $h^{d} \in H Z(G) H^{b} \leq G$ for every $d \in A$. Thus $h \in \cap\left[H Z(G) H^{b}\right]^{g}$, where $g$ ranges over the elements of $G$. This intersection is a normal subgroup of $G$ and we denote it by $N(b)$ (thus $N(b)$ is the core of $H Z(G) H^{b}$ in $G$ ). From Lemma 2.1 it follows that $H Z(G) H^{b}=H N(b)$.

If we write $a b=k f$, where $k \in H$ and $f \in A$, then likewise $k \in N(a)$, where $N(a)$ is naturally the core of $H Z(G) H^{a}$ in $G$. Clearly, $N(a) \geq K Z(G)$ for every $a \in A, a b \in A N(b)$ and also $a b \in N(a) A$.

If $|N(a) \cap H| \geq p^{4}$, then $H N(a) / N(a)$ is cyclic and by Theorem 2.4, $G^{\prime} \leq H N(a)=H Z(G) H^{a}$. We now consider the conjugates $H Z(G) / K Z(G)$ and $H^{a} Z(G) / K Z(G)$ and write $H Z(G) \cap H^{a} Z(G)=L Z(G)$, where $L \leq H$. From Lemma 2.9 it follows that $L Z(G)$ is larger than $K Z(G)$. Now $L Z(G)=$ $Z\left(H Z(G) H^{a}\right)$ and as $H Z(G) H^{a}$ is normal in $G$, it follows that the core of $H Z(G)$ is larger than $K Z(G)$, a contradiction. Thus we may assume that $|N(a) \cap H| \leq p^{3}$ for every $a \in A$.

Then consider the case that $a b=c h, N(a) \cap H \neq N(b) \cap H$ and $\mid N(a) \cap$ $H\left|=p^{3}=\right| N(b) \cap H \mid$. By Theorem 2.4, it follows that $G^{\prime} \leq H N(a) N(b)=$ $H Z(G) H^{a} H^{b}$. By Lemma 3.2, $h \in Z\left(H Z(G) H^{a} H^{b}\right) \leq N_{G}(H)=H \times Z(G)$. As $Z\left(H Z(G) H^{a} H^{b}\right)$ is normal in $G$, we conclude that $h \in K$. Thus $a b \in A K$.

If $a b=c h$ and $N(a) \cap H=K$ or $N(b) \cap H=K$, then $a b \in K A$ or $a b \in A K$. By Lemma 3.1, $A Z(G) \subseteq A$ and as $K Z(G)$ is normal in $G$, we conclude that $A K=K A$ is a subgroup of $G$. Thus we see that $A^{2} \subseteq A K<G$, contradicting $\langle A\rangle=G$.

3) Now assume that $K \cong C_{p} \times C_{p} \times C_{p}$. In part two of the proof we showed that $|N(a) \cap H| \leq p^{3}$ for every $a \in A$. As $N(a) \cap H \geq K$, we must have $N(a) \cap H=K$ for every $a \in A$. But then $A^{2} \subseteq A K<G$, a contradiction.

Let $Q$ be a loop and $M(Q)^{\prime} \leq N_{M(Q)}(I(Q))=I(Q) \times Z(M(Q))$. This is equivalent of $Q$ being centrally nilpotent of class at most two (see Bruck [1], also Section 6 in [11]). By combining Theorem 2.10 with Theorem 3.3 we thus get 
Theorem 3.4. Let $Q$ be a finite commutative IP-loop and let $I(Q)$ be an elementary abelian group of order $p^{5}$. Then $Q$ is centrally nilpotent of class at most two.

\section{References}

[1] Bruck,R.H., Contributions to the theory of loops, Trans. Amer. Math. Soc. 60 (1946), $245-354$.

[2] Csörgö,P., On connected transversals to abelian subgroups and loop theoretical consequences, Arch. Math. (Basel) 86 (2006), no. 6, 499 - 516.

[3] Csörgö, P., Abelian inner mappings and nilpotency class greater than two, European J. Combin. 28 (2007), no. 3, 858 - 867.

[4] Drápal,A., Vojtěchovský,P., Explisit constructions of loops with commuting inner mappings, European J. Combin. 29 (2008), no. 7, 1662 - 1681.

[5] Kepka,T., Niemenmaa,M., On multiplication groups of loops, J.Algebra 135 (1990), $112-122$.

[6] Kepka,T., Niemenmaa,M., On connected transversals to abelian subgroups, Bull. Australian Math. Soc. 49 (1994), 121 - 128.

[7] Leppälä,E., Niemenmaa,M., On finite commutative loops which are centrally nilpotent, Comment. Math. Univ. Carolin. 56,2 (2015), 139 - 143.

[8] Niemenmaa,M., Finite loops with nilpotent inner mapping groups are centrally nilpotent, Bull. Australian Math. Soc. 79 (2009), 109 - 114.

[9] Niemenmaa,M., On finite commutative IP-loops with elementary abelian inner mapping groups of order $p^{4}$, Comment. Math. Univ. Carolin. 51,4 (2010), $559-563$.

[10] Niemenmaa,M., On dihedral 2-groups as inner mapping groups of finite commutative inverse property loops, Comment. Math. Univ. Carolin. 59,2 (2018), 189 - 193.

[11] Niemenmaa,M., Rytty,M., Connected transversals and multiplication groups of loops, Quasigroups Related Systems 15 (2007), 95 - 107.

Department of Mathematical Sciences

University of Oulu, PL 3000

90014 Oulu, Finland

E-mail: markku.niemenmaa@oulu.fi 